\title{
A Recommended Biomechanical Model for Calculating Tension Angles of Muscles Working on Elbow Flexion
}

\author{
Mohamed Gaber Brekaa*, Yasser Atif Ghoraba**, Wael Mohamed Abd El-Kader***
}

\section{Introduction and Research Problem}

$\mathrm{M}$ otion analysis in studying the technical aspects of a skill aims at identifying the advantages and disadvantages of various ways of skill performance to establish training and teaching bases scientifically to guarantee the highest performance levels $(1: 8)$.

There is a close well-documented relation between athletic performance and motor analysis. Knowing this relation is necessary for biomechanical research and use of research results in sports training as well (2).

Biomechanical information is valuable for sports medicine as it shows injury mechanisms, epidemiology and prevention measures, in addition to exercises that may help or contradict with rehabilitation (3).

To perform biomechanical research, motor analysis labs, mechanical tests and biomechanical sports motor simulation devices are needed (4).

Biomechanical models of the human body are very important to understand the functional principles of human motion (5). It is a trial for imagining or presenting reality. In most cases, these models are much simpler than real counterparts (6).

Force is a very important biomechanical variable that affects motor performance in general. It is affected by force amount, point of effect and force direction identified by muscular tension angles. Changing any one of these variables leads to changes in the nature of force,

\footnotetext{
* Professor of Bio-Mechanics, Head of Kinesiology Dept, Faculty of Physical Education, Tanta University, Egypt.

** Assistant Professor, Kinesiology Dept, Faculty of Physical Education, Tanta University, Egypt.

*** Teacher, Ministry of Education, Egypt.
}

and consequently the nature of resultant motion. Tension angle is the angle between force line of work and level of moving limb bone (7).

After review of literature, the researchers concluded that none of the previous research works led to clear measurements for tension angles of various body muscles in each angle of joint during motion. In addition, there is no known device, for researchers, to measure these angles during motor performance phases.

This indicates the need for a biomechanical model for calculating muscular tension angles as tension angle for each muscle working on the joint changes according the change of joint angle during motion phases. This is of great importance for muscular motor analysis.

\section{Aim}

The current research aims at designing a biomechanical model for calculating muscular tension angles for muscle working on elbow flexion.

\section{Methods}

\section{Approach}

The researchers used the experimental approach.

\section{First phase (Design)}

Choosing a big skeleton, made of plastic, that is sturdy and naturally movable for lower arm

Choosing the right type of semi-muscles that is elastic and identifies the tension line between origin and fusion without any obstruction among muscles or the joint movement. A rubber cord was fixed using wax gun 
Semi-muscles were fixed from origin to fusion at Anatomy Department, Faculty of Medicine Tanta University to identify the tension line through elbow flexion as shown in table (1). Semi-muscles were colored differently to be differentiated.

Identifying the measuring tool for elbow angles on the model. This led to design a modified protractor (like a tension-meter) so that each edge is put over one of the joint bones to read the angle between them on the protractor accurately (fig. 1)

Identifying a mean for fixing the elbow joint on the required angle for the model. A mechanical device (a beech cuboids with two screws attached to fixers for each joint bone with a circular nut that moves the screw upwards and downwards to increase or decrease it length according to the required joint angle) (Fig. 1)

Table (1)

Working muscles during elbow flexion

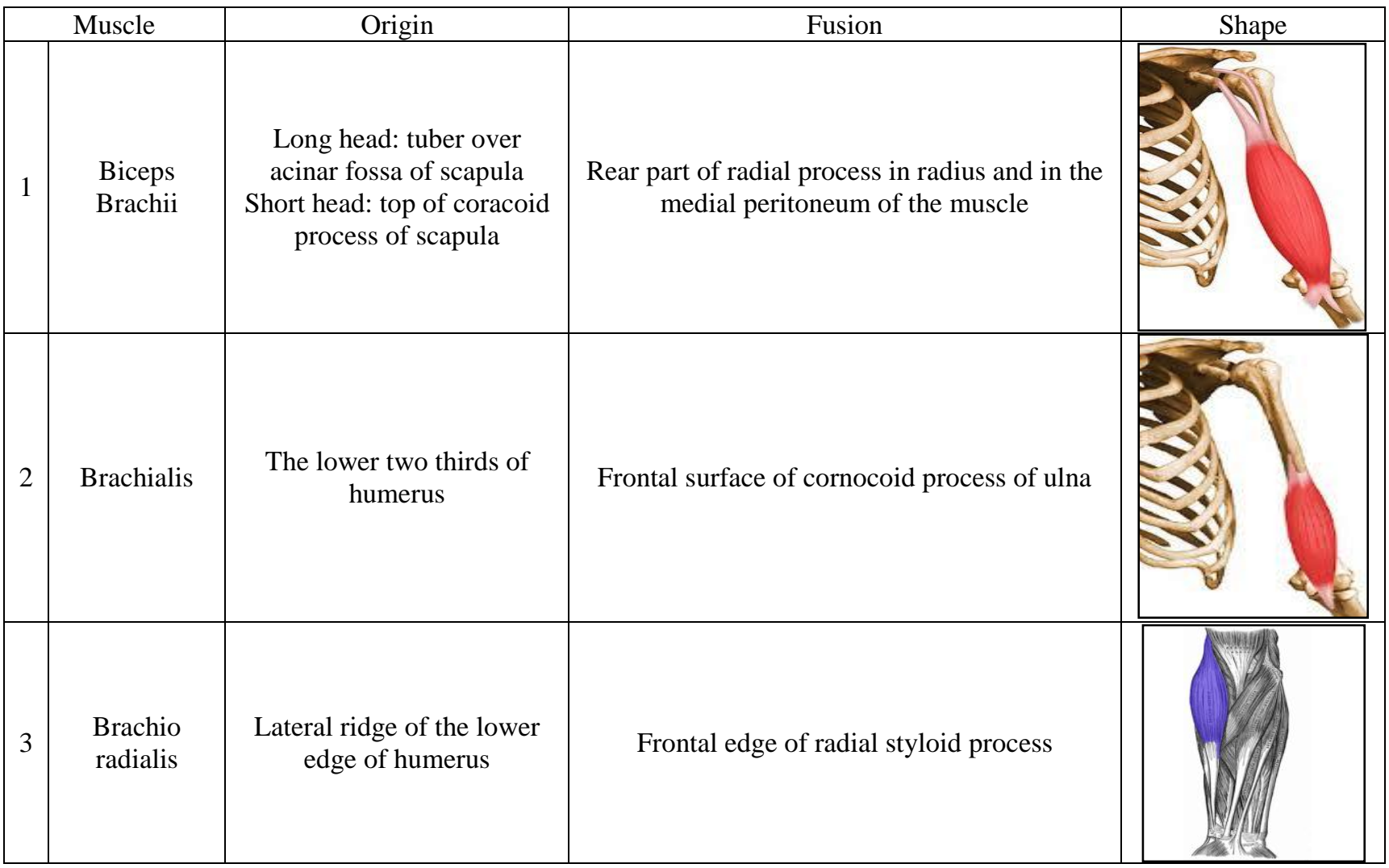

$(8,9,10 \& 11)$

Figure (1)

Tools for accurate simulation

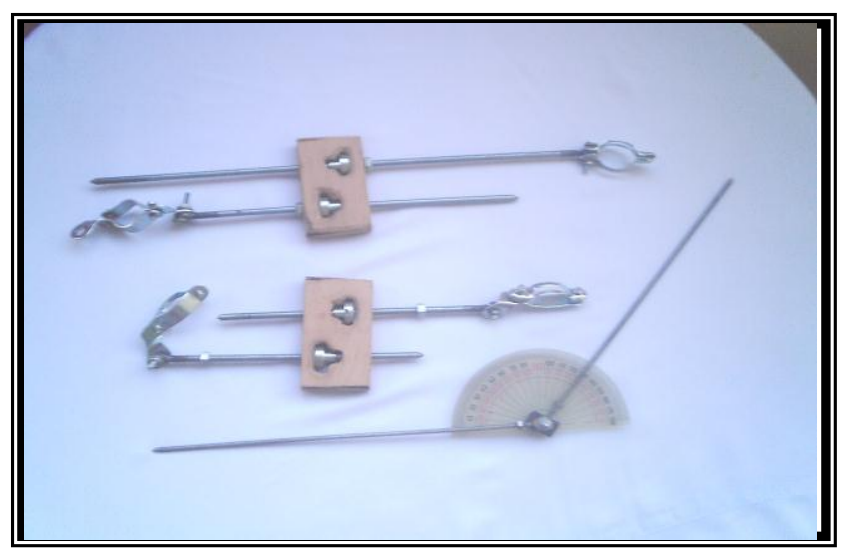




\section{Second phase (simulation and photography)}

After preparing the model, simulation begins with fixing elbow angle like its counterparts in natural movement according to the angles required for measuring muscular tension. Photography was applied to these positions.

\section{Procedures of photography}

1. Using a digital camera for facilitating photo input

2. Fixing the camera perpendicular to elbow joint and working muscles

3. Using a rear curtain with different color

4. Fixing numbers over the model that appear in each picture for cadre numbering

5. Using synthetic lights distributed to cover shadows

6. Shooting working muscles in these cadres to be discriminated through identifying its line of work, the moving bone and cadre number.( appendix 1,2)

\section{Third phase (measuring tension angles)}

- To measure tension angles for each position, these procedures were followed:

- Each photo was inputted into computer from camera memory card through a card reader to be transferred quickly with high resolution

- Photos were categorized according to cadre number in separate files

- Autocad 2002 software was used to measure tension angles for each muscle with high accuracy

\section{Tools and equipments}

- A full skeleton with holder rubber cords

- Wax gun

- Protractor for measuring joint angles

- Mechanical device for fixing angles

- Mechanical toolkit (screws - screw driver scissors - nuts - ...etc)

- Rear curtains

- Digital camera

- Card reader
- PC with Autocad 2002 software.

\section{Recommendations}

1. Using the recommended biomechanical model for analyzing muscular work and calculating tension angles of muscles working during elbow flexion

2. Using the recommended biomechanical model to estimate force amounts during elbow flexion.

3. Using the recommended biomechanical model as an aid to demonstrate the anatomy of muscles working on elbow flexion

4. Designing other models for various body joints to be used in motor analysis.

\section{References}

1. Heppe, Robert (1999): Biomechanics \& Motion Analysis, Article: what is motion analysis study? Biomechanical Solutions, P:1

2. Hochmout, Gerd (1999): Biomechanics and research methods for sports movements, 3rd ED (in German) (Gamal Abd El-Hameed \& Soliman Hasan translators) Markaz Al-Ketab Press, Cairo - Egypt. P: 24 (in Arabic)

3. Berequa, Mohamed G. \& Al-Sokkary Khairia (2002): Basics of sports biomechanics part one. Munshaat Al-Maaref, Alexandria - Egypt. P: 31-35 (in Arabic)

4. Cavanagh, Peter R.; Grabiner, Mark D. \& others (2005): Biomechanics, Learner research institute, department of biomechanical engineering, The Cleveland Clinic, LRI. P:1

5. Lindsay, Natalia (2001): Modeling of the shoulder mechanism, a report describing the development of 3D biomechanical model of the human shoulder complex. Queensland Institute of Technology, Brisbane, Australia. P:1

6. Whiting, William C. \& Zernicke, Ronald F. (1998): Biomechanics of musculoskeletal injury, Human Kinetics, USA. P:79

7. Hosam El-Din, T.; Abd El-Rasheed S.; Kamel M. \& Salah El-Din, W. (1998): Applied Kinesiology, part one, 1st Ed. Markaz Al-Ketab Press, Cairo - Egypt. PP:183-187 (in Arabic) 
8. De Graaff, Van M. (2001): Human anatomy, USA, WMC Brown Publishers PP:152-153

9. Tortora, Gerard J. (1997): Introduction to the human body, the essentials of anatomy and physiology, New York, Benjamin Coming. PP:169-170
10. Hendy, Mohamed F. (1991): Sports Anatomy. Dar Al-Fikr Al-Araby, Cairo - Egypt. PP: 167-173 (in Arabic)

11. Abrahams, Hutching, Marks Jr (1998): McMinn's color atlas of human anatomy, 4th Ed, Mosby. PP:116-121 